\title{
ProEvaluator: Uma Ferramenta para Avaliação de Processos de Software
}

\author{
Juliana Moura Cavalcanti Xavier', Alexandre Marcos Lins de Vasconcelos ${ }^{2}$ \\ ${ }^{1}$ Serviço Federal de Processamento de Dados (SERPRO) \\ Av. Parnamirim, 295, CEP: 52060-000 - Recife - PE - Brasil \\ ${ }^{2}$ Centro de Informática - Universidade Federal de Pernambuco (UFPE) \\ Caixa Postal 7851 - CEP: 50732-970 - Recife - PE - Brasil \\ $\{$ jmcx, amlv\}@cin.ufpe.br
}

\begin{abstract}
In December 2003, SOFTEX (Association for Promoting Brazilian Software Excellence) launched the MPS.BR (the Model for Brazilian Software Process Improvement). The MA-MPS (MPS.BR Evaluation Method) was created to check if the organizations meet the requirements of such model. This paper presents a tool to support the activities described in the MA-MPS method. The tool allows the record of the process execution evidences, the attribution of concepts to the process expected results and, from that point, calculates automatically the maturity level of the organization and releases a final report with the evaluation results.
\end{abstract}

Resumo. Em dezembro de 2003, a SOFTEX (Associação para Promoção da Excelência do Software Brasileiro) lançou o MPS.BR (Modelo para Melhoria de Processo de Software Brasileiro). Para verificar se as organizações atendem aos requisitos do modelo criado, foi definido o MA-MPS (Método de Avaliação do MPS.BR). Este artigo apresenta uma ferramenta para apoiar as atividades descritas no método MA-MPS. A ferramenta permite o cadastro de evidências da execução do processo, atribuição de conceitos aos resultados esperados dos processos e de seus atributos e, a partir daí, calcula automaticamente o nível de maturidade da organização e emite um relatório final com os resultados da avaliação.

\section{Introdução}

Avaliação de Processo de Software tem sido um método popular e largamente utilizado para garantir a produção de software de qualidade. Esta atividade tem sido realizada por organizações que desejam melhorar seus processos de software e, conseqüentemente, seus produtos de software.

Uma avaliação de processos de software produz uma quantidade enorme de dados associados à avaliação. Assim, a manipulação e análise desses dados são aspectos importantes na avaliação de processos. Logo, o uso de uma ferramenta para manipular os dados da avaliação se torna imprescindível. Segundo [HUNTER, 1997], os principais aspectos de manipulação de dados associados à avaliação de processos de software são: a captura, o armazenamento, a visualização e análise dos dados. 
As ferramentas de avaliação de processos de software [WALKER, 1995] [FABBRINI, 2003] costumam mostrar um resumo dos principais resultados da avaliação de uma forma gráfica. Tais ferramentas permitem ao usuário visualizar os dados da avaliação e, assim, identificar os pontos fracos do processo e priorizar as atividades de melhoria.

A ferramenta proposta por esse trabalho visa automatizar algumas atividades do Método de Avaliação MPS.BR, o MA-MPS. Hoje em dia, se alguma empresa deseja automatizar o processo de avaliação, precisa criar sua própria ferramenta, pois as que existem estão disponíveis apenas para consultorias. A ferramenta ProEvaluator ficará disponível gratuitamente para download pela Internet.

O artigo está organizado em 5 seções. A seção 2 apresenta os trabalhos relacionados, ou seja, as ferramentas de avaliação de processos de software mais conhecidas. A seção 3 examina os principais métodos de avaliação, focando no Método de Avaliação do MPS.BR, o MA-MPS. A seção 4 explica a proposta da ferramenta ProEvaluator, que é o foco deste trabalho. A seção 5 mostra uma análise crítica da ferramenta. Por fim, a seção 6 conclui o artigo e apresenta os possíveis trabalhos futuros.

\section{Trabalhos Relacionados}

A ferramenta PISA provê um suporte automático para as atividades de avaliação de processos de software baseadas na norma ISO/IEC 15504 [FABBRINI, 2003]. Ela oferece um apoio às atividades de coleta/validação de dados e fornecimento de notas aos atributos de processo. De acordo com a metodologia automatizada por essa ferramenta, a avaliação de processos é dividida em quatro partes: preparação, coleta de dados, avaliação e emissão de relatórios. A fase de preparação inclui atividades de registro de dados da organização, configuração de pesos das características da avaliação e registro do escopo da avaliação. A fase de coleta de dados contém a atividade de preenchimento de checklists. A fase de avaliação inclui as atividades de análise de práticas base e práticas de gerenciamento automaticamente. A última fase consiste na emissão do relatório final de avaliação.

A ferramenta para avaliação de processos SEAL [WALKER, 1995] está disponível na Internet gratuitamente. Ela provê uma abordagem sistemática para a realização de avaliações baseadas no modelo de avaliação definido pela norma ISO/IEC 15504. É possível criar várias instâncias de avaliação e para cada uma delas, por meio de uma interface gráfica, registrar o nível alcançado dos produtos de trabalho, práticas base e práticas de gerenciamento. Uma vez que esses níveis são determinados, a ferramenta avalia automaticamente os atributos de processo. É possível aumentar ou diminuir os atributos de processo, práticas base, práticas de gerenciamento e produtos de trabalho, por isso essa ferramenta é adaptável a pequenas evoluções da norma ISO/IEC 15504, versão relatório técnico.

O CORE-KM (Customizable Organizational Resources Environment with Knowledge Management) é um ambiente customizável para gerência de conhecimento em diferentes organizações, capaz de apoiar seus processos organizacionais [GALOTTA, 2004]. A infra-estrutura base do CORE-KM traz uma série de ferramentas 
genéricas que podem ou não estar nos ambientes customizados, em função da necessidade de cada organização.

O Ambiente de Apoio a Avaliações MPS pode ser instanciado a partir do CORE-KM. Este ambiente tem o conhecimento comum MPS e os templates de artefatos requeridos para a execução do processo [MURADAS, 2006]. A Instituição Avaliadora precisa então cadastrar suas próprias informações, bem como definir sua interface. A ferramenta é utilizada para apoiar uma Instituição Avaliadora na realização de avaliações MPS. Os principais objetivos desse ambiente é o apoio à execução das atividades de planejamento do processo de avaliação MPS, a gerência das atividades das Instituições Avaliadoras e a disseminação das experiências vividas pelos avaliadores durante a realização das avaliações segundo o método de avaliação MPS.BR.

O Ambiente de Apoio a Avaliações MPS é composto por 3 ferramentas específicas. A primeira trata do apoio ao processo de avaliação MPS propriamente dito. A segunda trata do cadastro e consulta dos profissionais da Instituição Avaliadora com suas características. A última é uma agenda para se cadastrar atividades e consultar a disponibilidade dos profissionais para realizar as avaliações.

Appraisal Assistant é uma aplicação de software desenvolvida pelo Software Quality Institute, da Griffith University, para dar apoio à avaliação da capacidade do processo ou maturidade organizacional. Sua abordagem é consistente com os requisitos do Processo de Avaliação da ISO/IEC 15504 e os requisitos de avaliação do CMMI. Diferente de outras ferramentas de avaliação de processos, Appraisal Assistant possui uma abordagem dirigida a evidências para registrar a informação gerada durante a avaliação. Ela provê suporte ao método de avaliação SCAMPI e ao método genérico definido pela Norma ISO/IEC 15504. Ou seja, é possível expressar resultados de uma avaliação CMMI como perfis de processo padrão ISO/IEC 15504. A ferramenta também facilita a geração de relatórios da avaliação.

Nas avaliações MPS.BR, os avaliadores não utilizam nenhuma ferramenta automatizada, além de uma planilha de indicadores em Excel. Tal planilha contém os processos que vão ser avaliados e os seus respectivos resultados esperados. Ao final da avaliação, a planilha contém todos os resultados esperados para os processos e seus atributos que estão sendo avaliados. Para cada resultado esperado, evidências que comprovam que o resultado foi alcançado são inseridas. Para cada evidência, identifica-se a fonte da mesma e os projetos aos quais ela pertence. Caso a evidência não pertença a projetos específicos, e sim à organização toda, informa-se que se trata de uma evidência organizacional.

O avaliador também informa um conceito para cada projeto e cada resultado esperado, através da análise das evidências do projeto. Baseado nos conceitos informados, calcula-se o conceito geral do resultado esperado. Se todos os resultados esperados de um processo forem satisfatórios, então o processo como todo é dito como satisfeito. Caso todos os processos sejam caracterizados como satisfeitos, conclui-se que a organização atingiu aquele nível organizacional almejado. 


\subsection{Comparação entre as Ferramentas de Avaliação}

A seguir encontra-se uma tabela com o resumo geral das principais características das ferramentas descritas.

Tabela 1.1 Características das Ferramentas de Avaliação

\begin{tabular}{|c|c|c|c|c|c|}
\hline Ferramenta & & 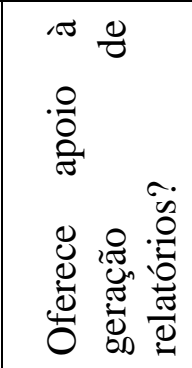 & 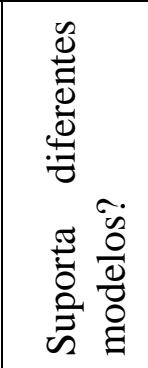 & 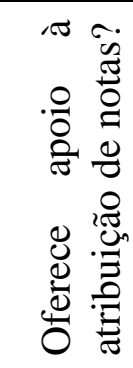 & 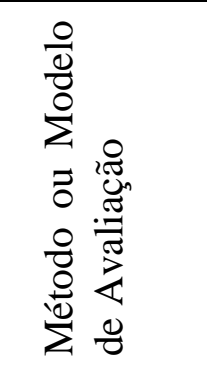 \\
\hline PISA & Não & Sim & Sim & Sim & $\begin{array}{l}\text { ISO/IEC } \\
15504\end{array}$ \\
\hline SEAL & Sim & Sim & Não & Sim & $\begin{array}{l}\text { ISO/IEC } \\
15504\end{array}$ \\
\hline $\begin{array}{l}\text { Ambiente de Apoio às } \\
\text { Avaliações MPS.BR }\end{array}$ & Sim & Sim & Não & Não & MA-MPS \\
\hline Appraisal Assistant & Sim & Sim & Sim & $\operatorname{Sim}$ & $\begin{array}{l}\text { SCAMPI e } \\
\text { ISO/IEC } \\
15504\end{array}$ \\
\hline $\begin{array}{l}\text { Planilha de Avaliação } \\
\text { MPS.BR }\end{array}$ & Sim & Não & Não & Não & MA-MPS \\
\hline
\end{tabular}

Como podemos observar, a ferramenta que possui uma maior cobertura em relação às características comuns de uma ferramenta de avaliação é a Appraisal Assistant. Ela é uma ferramenta gratuita que suporta diferentes modelos de avaliação. Ela oferece apoio à avaliação baseada no método SCAMPI e no método baseado na Norma ISO/IEC 15504, porém ela não fornece apoio ao MA-MPS. Essa ferramenta também possui uma gama de tipos de relatórios que podem ser emitidos no fim da avaliação. As ferramentas PISA e SEAL são baseadas no modelo ISO 15504, oferecem suporte à atribuição de notas e geração de relatórios de avaliação. Além disso, a ferramenta SEAL está disponível gratuitamente na Internet, porém, não é adaptável a diferentes modelos. Já a ferramenta PISA é adaptável a pequenas evoluções do modelo ISO 15504. O Ambiente de Apoio às Avaliações MPS.BR está disponível gratuitamente 
e fornece suporte à fase de planejamento do método MA-MPS, mas não contempla às fases de avaliação e geração de relatório final. A planilha de avaliação MPS.BR é disponibilizada gratuitamente pela SOFTEX apenas para as empresas que serão avaliadas oficialmente. Por não ser uma ferramenta automatizada, a planilha não fornece suporte a relatórios e só pode ser utilizada para avaliar processos baseados no modelo MPS.BR. A proposta desse trabalho, que é a ferramenta ProEvaluator, é gratuita, oferece suporte a relatórios, porém não é adaptável a diferentes modelos. No estágio atual, ela automatiza apenas parte do Método de Avaliação MPS.BR.

\section{Métodos de Avaliação}

Os métodos de avaliação definem o procedimento de avaliação de processos de software. O método mais comum é o Standard CMMI Appraisal Method for Process Improvement - SCAMPI [CMU/SEIa, 2002]. Ele é o método de avaliação descrito no modelo de referência CMMI. O método de avaliação do MPS.BR é o MA-MPS [MPS.BR, 2006b]. Ele orienta a execução de uma avaliação em conformidade ao Modelo de Referência MPS.BR.

O Appraisal Requirements for CMMI, versão 1.1 - ARC V1.1 foi publicado pelo CMMI Product Team em 2001 [CMU/SEId, 2001]. Ele define os requisitos para os métodos de avaliação de processo que têm como base de melhoria o modelo CMMI. O ARC V1.1 define um conjunto de classes de avaliação baseadas em aplicações típicas de métodos de avaliação. Ele identifica três classes de métodos de avaliação, denominados Classe A, Classe B e Classe C.

Os métodos da Classe A devem satisfazer todos os requisitos do ARC V1.1. Esta é a única classe de métodos que pode fornecer graduação para análise comparativa (benchmarking). Os métodos dessa classe exigem que sejam aplicadas 3 fontes de dados na avaliação: entrevista, aplicação de instrumentos e revisão de documentação.

Os métodos da Classe B devem satisfazer um subconjunto dos requisitos do ARC V1.1. Exigem apenas duas das três fontes de dados (entrevista, aplicação de instrumentos e revisão de documentação) dos métodos de classe $\mathrm{A}$, sendo que uma destas fontes deve ser a entrevista. Além disso, não fornecem graduação e são indicados para organizações que estão começando as atividades de melhoria de processo utilizando modelos CMMI.

Os métodos da Classe $\mathrm{C}$ devem satisfazer apenas um subconjunto dos requisitos dos métodos de Classe B. Exigem apenas uma das três fontes de dados dos métodos de Classe A. A validação das observações e o mecanismo de consenso entre os membros da equipe são requisitos opcionais desta classe de métodos. Este tipo de avaliação deve ser, provavelmente, mais utilizado quanto o objetivo é dar uma "rápida olhada" ou, para os casos de auto-avaliações periódicas, feitas por grupos de projetos ou de suporte.

O Standard CMMI Appraisal Method for Process Improvement - SCAMPI V1.1 é um método de avaliação compatível com os requisitos definidos pelo ARC V1.1 e atende avaliações conduzidas de acordo com a norma ISO/IEC TR 15504. Ele fornece a graduação da maturidade de processo em relação aos modelos CMMI, satisfaz todos os requisitos de métodos Classe A e é composto de três fases e onze processos. 
O Método de Avaliação do MPS.BR (MA-MPS) está em conformidade com a Norma Internacional para avaliação de processos de software ISO/IEC 15504 [ISO/IEC 15504-3, 2004]. Ele permite a avaliação objetiva dos processos de software de uma organização [MPS.BR, 2006b]. O MA-MPS pode ser aplicado a organizações de diversos tamanhos e diferentes domínios de aplicação. O resultado final da aplicação do método é a atribuição de um nível de maturidade para a organização com base no resultado da avaliação.

A equipe de avaliação do MA-MPS é composta de 3 a 8 pessoas, sendo um avaliador líder, no mínimo, um avaliador adjunto e um técnico da empresa. A duração da avaliação varia de 2 a 4 dias. A validade de uma avaliação MPS é de 3 anos. Após esse período, a organização deve se submeter novamente à avaliação de seus processos.

O processo de avaliação define um conjunto de atividades realizadas para verificar a maturidade da unidade organizacional na execução de seus processos de software. Cada avaliação tem um patrocinador que financia a realização da mesma, ou seja, ele é responsável pelos gastos necessários para execução da avaliação. Para realização de uma avaliação o patrocinador deve, inicialmente, selecionar uma Instituição Avaliadora dentre as credenciadas pela SOFTEX. Este processo se encerra com o registro da avaliação na base de dados confidencial da SOFTEX.

Através da execução do processo de avaliação obtemos dados e informações que caracterizam os processos de software da organização. Também é determinado o grau em que os resultados esperados são alcançados e o nível em que os processos atingem seu propósito.

O processo de avaliação é composto de quatro sub-processos principais. O resultado da execução do sub-processo "Contratar Avaliação" é estabelecer um contrato para realização da avaliação MPS. Essa contratação pode ser feita de três formas. A organização pode estabelecer um contrato diretamente com uma ou mais Instituições Avaliadoras credenciadas pela SOFTEX. Outra opção é a organização entrar em contato com a SOFTEX e solicitar uma avaliação. Neste caso, a SOFTEX pode encaminhar para uma Instituição Avaliadora ou ser a contratada, designando avaliadores credenciados para realizar a avaliação de forma independente das Instituições Avaliadoras. A última forma de solicitar uma avaliação é uma empresa diferente da unidade organizacional a ser avaliada solicitar uma avaliação de terceira parte para fins de contrato.

A segunda fase do processo de avaliação é "Preparar para Realização da Avaliação". A organização recebe o template do Plano de Avaliação da SOFTEX e preenche o mesmo com informações sobre a organização. Durante essa fase, a organização também deve assinar um Acordo de Confidencialidade e agendar a avaliação inicial.

A terceira fase do processo consiste em "Realizar a Avaliação". O propósito desse sub-processo é treinar a equipe, conduzir a avaliação MPS e comunicar seus resultados à unidade organizacional avaliada. É durante essa fase que são analisadas as evidências diretas e indiretas, são realizadas as entrevistas e atribuídos conceitos aos resultados esperados dos processos. Depois, é feita uma avaliação nos processos selecionados e o nível organizacional é obtido e reportado para a organização. 
A última fase do processo é responsável por "Documentar os resultados da avaliação". O propósito desse sub-processo é elaborar o relatório da avaliação, enviá-lo ao patrocinador da avaliação e à SOFTEX, que é responsável por inserir os dados da avaliação em sua base de dados e divulgar o resultado em seu site. Um detalhamento maior sobre o processo de avaliação do MA-MPS pode ser obtido através do Guia de Avaliação [MPS.BR, 2006b].

Os métodos MA-MPS e SCAMPI são bem semelhantes. Ambos definem uma fase de Planejamento de Avaliação, pregam o consenso da equipe para definir o nível organizacional, fazem uma apresentação final dos resultados apontando os pontos fortes e oportunidades de melhoria e divulgam o resultado final da avaliação apenas com o consentimento do patrocinador. A principal diferença entre esses métodos consiste no tipo de graduação que os mesmos fornecem. Os avaliadores que seguem o método MAMPS avaliam o nível de maturidade da organização enquanto os avaliadores do SCAMPI analisam o nível de maturidade da organização e/ou de capacidade de seus processos.

\section{ProEvaluator}

A proposta da ferramenta ProEvaluator é automatizar algumas atividades do método de avaliação MPS.BR. Ela pode ser usada nas organizações antes ou durante a avaliação oficial MPS.BR. Ou seja, essa ferramenta pode ser utilizada para as organizações se auto-avaliarem, podendo identificar seus pontos fracos e fortes; ou durante a avaliação oficial MPS.BR para que os avaliadores possam analisar os processos da organização e então atribuir conceitos aos respectivos resultados esperados. No fim da avaliação, a ferramenta exibe um relatório contendo o nível de maturidade do processo organizacional, de acordo com os níveis de maturidade descritos no modelo de referência MPS.BR.

Baseado no estudo dos requisitos das principais ferramentas de avaliação de processos do mercado [FABBRINI, 2003] [WALKER, 1995] [LIANG, 2007] [MURADAS, 2006] e nas entrevistas realizadas com um avaliador oficial do MPS.BR, foram extraídos os seguintes requisitos para ferramenta ProEvaluator:

- Gratuidade: a ferramenta será totalmente gratuita e estará disponível para download na Internet;

- Adaptável a diferentes domínios: a ferramenta poderá ser usada para avaliação de processos em organizações de diferentes tamanhos e domínios;

- Baseada no método de avaliação do MPS.BR: a ferramenta visa automatizar as atividades do processo de avaliação do MPS.BR, versão 1.0.

- Suporte a relatórios: no final da avaliação será permitido ao usuário emitir um relatório final com o resultado da avaliação. Durante a execução da avaliação, relatórios parciais poderão ser emitidos.

O ambiente de apoio às avaliações MPS definido por [MURADAS, 2006] oferece suporte a fase de planejamento da avaliação; no entanto, não oferece suporte às 
demais fases da avaliação. O objetivo da construção da ferramenta ProEvaluator é automatizar as fases de "Realização da Avaliação" e "Documentação dos Resultados", descritas no processo de avaliação do MPS.BR.

A Figura 1 representa o modelo de casos de uso da ferramenta ProEvaluator, que fornece uma visão estática das funcionalidades da mesma. Podemos observar que existem 2 atores envolvidos no processo de avaliação: o usuário comum e o avaliador. O usuário pode criar ou abrir uma avaliação, inserir evidências diretas e indiretas para os resultados esperados, consultar o conceito dos processos, gerar os gráficos e o relatório final da avaliação. $\mathrm{O}$ avaliador é um usuário e além de todas as funcionalidades permitidas para o usuário também pode inserir afirmativas que são obtidas através das entrevistas, analisar os resultados esperados, atribuindo conceitos a eles e alterar os conceitos dos processos, se julgar necessário. Depois que o avaliador analisa todos os resultados esperados, o sistema calcula automaticamente o conceito para o processo.

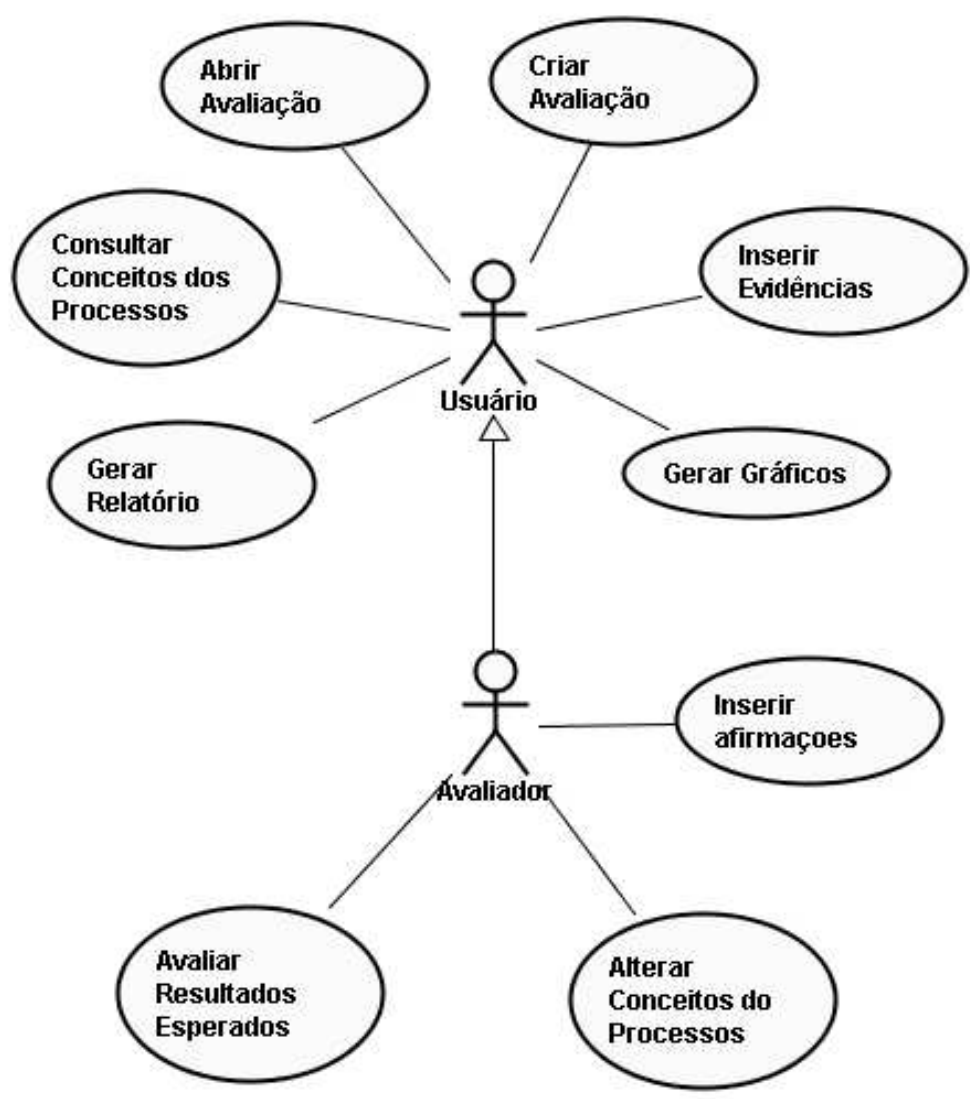

Figura 1. Modelo de Casos de Uso da ProEvaluator

Para criar uma avaliação é necessário fornecer o nome da mesma, os projetos que serão avaliados e o nível almejado pela Organização. A partir daí a avaliação já foi criada e pode ser reaberta através da ferramenta sempre que necessário. Por isso, no início da execução da avaliação, o usuário precisa tomar uma decisão: criar ou abrir uma avaliação já existente. A partir daí é exibida uma tela contendo as informações da 
avaliação, os processos que serão avaliados, os projetos e o nível almejado. O próximo passo é escolher o processo onde serão cadastradas as evidências do processo da Organização. O processo é exibido com seus resultados esperados e seus atributos de processo. O avaliador deve apenas selecionar o resultado que deseja cadastrar a evidência, escolher o tipo de evidência (Direta, Indireta ou Afirmativa) e realizar o cadastro. Ele deve realizar essa operação para todos os resultados esperados dos processos e de seus atributos. Após cadastrar as evidências, o avaliador procede com a atribuição de conceitos para os resultados esperados dos processos e de seus atributos. Nessa hora, ele pode descrever os pontos fortes e oportunidades de melhoria do processo da Organização. Essas informações estarão presentes no relatório final da avaliação. Depois que todos os resultados esperados de um processo forem avaliados, o sistema calcula automaticamente o conceito do mesmo (vide Figura 2). Esse conceito pode ser: Satisfeito, Não Satisfeito ou Fora de Escopo, conforme critérios definidos no MA-MPS.

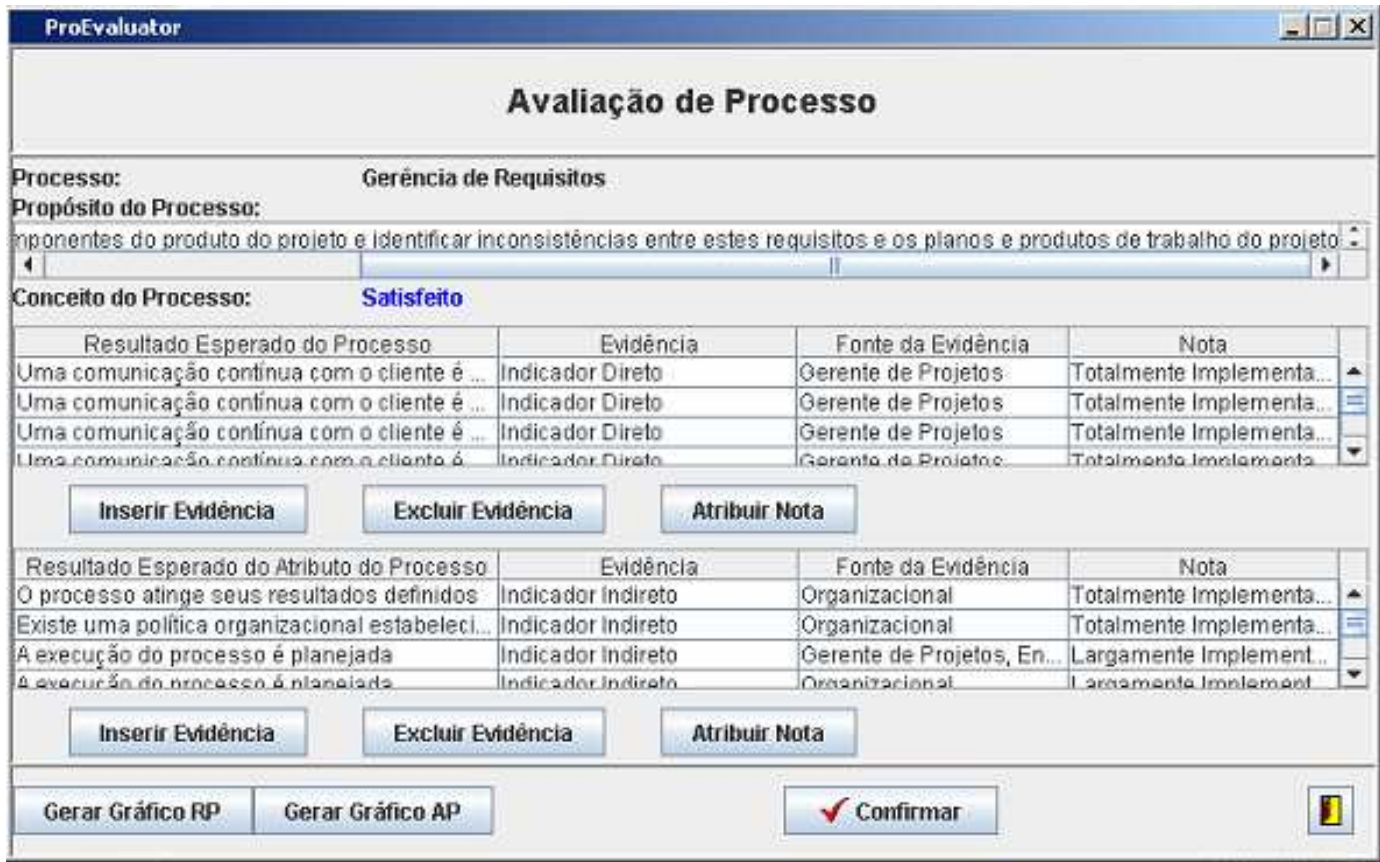

Figura 2. Avaliação de Processo na Ferramenta ProEvaluator

Se o avaliador não concordar com a nota gerada automaticamente pela ferramenta, ele pode alterá-la. A partir daí, o usuário pode gerar o gráfico dos resultados do processo e de seus atributos. Para finalizar a avaliação, é necessário criar o relatório final da mesma. Tanto o usuário comum quanto o avaliador podem realizar essa operação.

A ferramenta foi desenvolvida utilizando a modelagem orientada a objetos e a linguagem Java. Ela está dividida em três camadas: camada de negócio (classes básicas, cadastros e fachada), camada de dados (classes de persistência) e camada de apresentação (interfaces gráficas). Essa arquitetura foi projetada visando facilitar a manutenção evolutiva da ferramenta. Atualmente, ela está sendo evoluída para suportar outros métodos de avaliação. 


\section{Análise Crítica}

Para verificar se a ferramenta atendia aos requisitos especificados, foi necessário analisá-la do ponto de vista de quem iria utilizá-la na prática: os profissionais de avaliação de processos. Utilizamos um questionário com perguntas sobre o perfil do entrevistado e as principais características da ferramenta. Este questionário teve três objetivos principais: verificar se a ferramenta estava aderente ao método de avaliação do MPS.BR, verificar se a ferramenta poderia ser utilizada numa avaliação interna da organização, antes da avaliação MPS.BR oficial e verificar a usabilidade da ferramenta.

Em relação ao perfil do entrevistado foram feitas perguntas sobre o seu papel na organização, experiência em desenvolvimento de sistemas, nível de conhecimento em Engenharia de Software, nível de conhecimento em Modelos de Qualidade, nível de conhecimento em Métodos de Avaliação, experiência prática com avaliação de processo, se já utilizou ferramentas de avaliação e, por fim, se possui alguma certificação MPS.BR.

Em relação à avaliação da ferramenta foram feitas perguntas sobre o apoio que ela oferece ao cadastro de evidências, sua aderência ao MA-MPS, adequação em préavaliação, adequação numa avaliação oficial MPS.BR, exibição dos resultados, usabilidade e, por fim, os entrevistados fizeram observações gerais sobre a ferramenta ProEvaluator.

Houve três apresentações presenciais da ferramenta (uma a alguns participantes do Simpósio Brasileiro de Engenharia de Software 2007 e outras duas a alguns profissionais de duas empresas desenvolvedoras de software, uma pública e uma privada). Após cada apresentação, o questionário foi respondido e devolvido para análise. Em todas as apresentações, inicialmente foi feita uma palestra sobre a ferramenta demonstrando o contexto onde ela estava inserida, seus requisitos e principais funcionalidades. Posteriormente, foi feita uma demonstração da ferramenta em execução criando uma avaliação e analisando seus resultados. A partir daí, foi iniciada a discussão com perguntas e esclarecimentos sobre a ferramenta. Para concluir, foram distribuídos os questionários para os ouvintes da apresentação e recolhido o resultado da análise. O SBQS foi escolhido devido à alta disponibilidade de profissionais peritos no modelo MPS.BR. Foi uma ótima oportunidade de reunir profissionais e pesquisadores para discutir os requisitos de uma ferramenta de avaliação de processos de software. A empresa pública foi escolhida devido à elevada quantidade de profissionais certificados no modelo MPS.BR. Além disso, essa organização tem a intenção de realizar uma avaliação MPS.BR até o fim de 2008. A outra empresa foi escolhida por estar se preparando para uma avaliação de processos de software baseada no modelo CMMI. Além dessas apresentações, um manual de uso da ferramenta foi enviado via e-mail a alguns profissionais de tecnologia da informação e em seguida, após lerem o manual, o questionário foi respondido e devolvido também via e-mail.

Ao todo, foram respondidos 25 questionários, distribuídos nos papéis de Gerente de Qualidade, Analista, SQA e Desenvolvedor. As apresentações presenciais somaram um total de 17 espectadores e as avaliações "à distância" somaram 8 avaliadores.

Esta abordagem está longe de ser a ideal, tanto pelo número de respostas obtidas, como por não ser uma aplicação prática da ferramenta. Entretanto, ela nos 
permite ter uma idéia da aderência da ferramenta ao método MA-MPS, pois leva em conta a experiência de profissionais com diferentes perfis.

\subsection{Análise dos Resultados}

A primeira parte dos questionários teve como objetivo traçar um perfil dos entrevistados, do ponto de vista da experiência em avaliação de processos de software e do conhecimento em modelos e métodos de avaliação. Para isso, foram elaboradas perguntas sobre a experiência técnica, em engenharia de software, em processo de desenvolvimento e o conhecimento prévio no método de avaliação do MA-MPS.

Apesar da maioria dos entrevistados possuírem um bom conhecimento teórico dos métodos de avaliação, eles não possuem experiência prática em avaliação de processos. Mais de $50 \%$ dos entrevistados não possuem nenhum conhecimento prático nesta atividade (vide Figura 3).

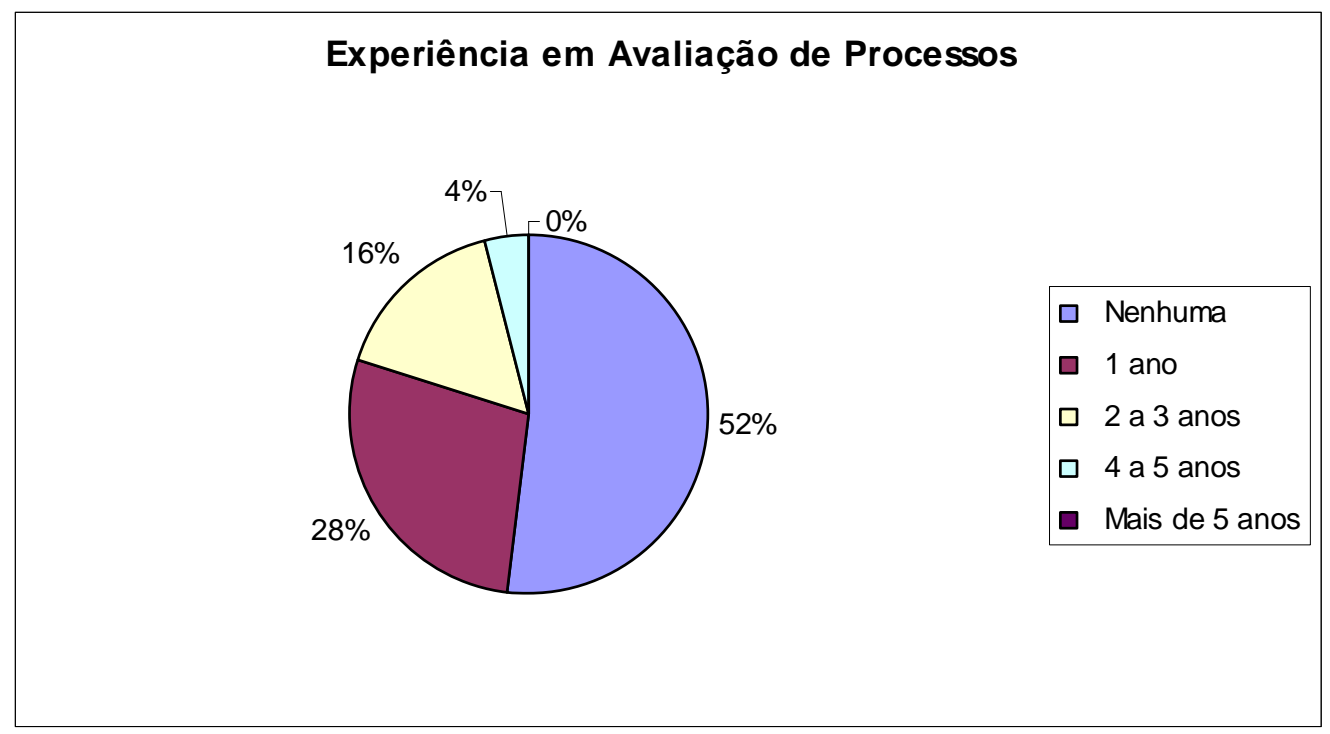

Figura 3. Experiência em Avaliação de Processos

A segunda parte do questionário teve como objetivo avaliar as funcionalidades da ferramenta ProEvaluator. A Figura 4 apresenta a adequação da ferramenta para uma avaliação anterior à avaliação oficial MPS.BR, ou seja, a uma pré-avaliação. Mais de $80 \%$ dos entrevistados informaram que ela é adequada para ser utilizada numa préavaliação. Aqueles que viram a ferramenta em execução fizeram uma avaliação mais positiva do que os que avaliaram pela Internet. Porém, um percentual considerável do total de entrevistados informou que ela poderia ser utilizada parcialmente em conjunto com outras ferramentas para realização de uma pré-avaliação. Acreditamos que esse seja o uso mais adequado da ferramenta no momento. Ela pode ser perfeitamente utilizada numa pré-avaliação para diagnotiscar pontos fortes e oportunidades de melhoria no processo da organização. 


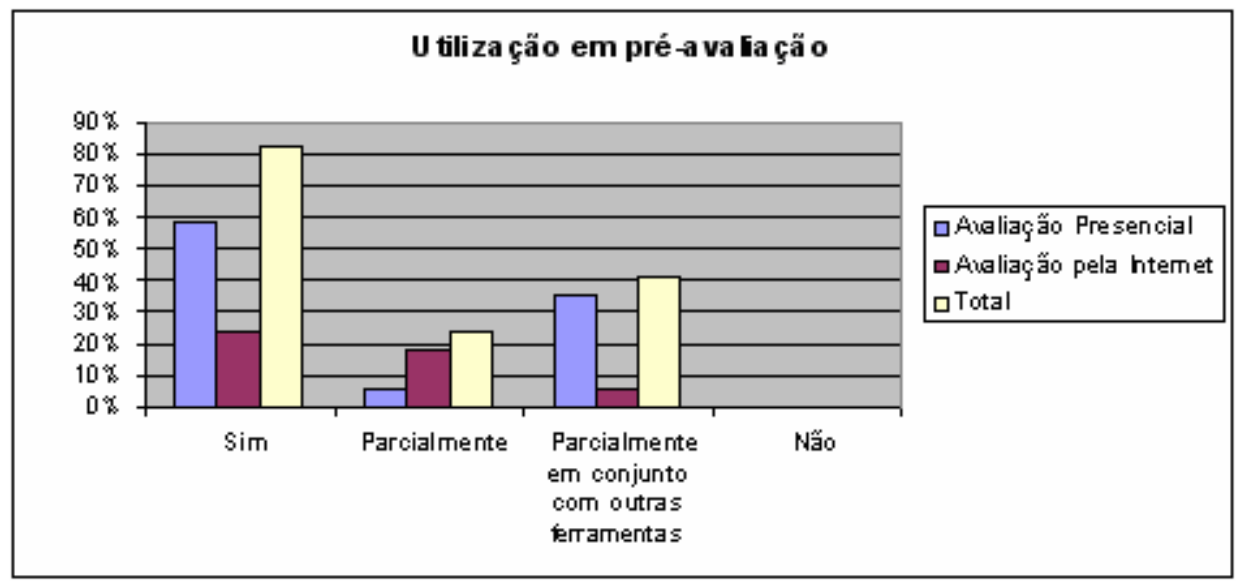

Figura 4. Utilização em pré-avaliação

\section{Conclusão}

O trabalho descrito teve por objetivo o desenvolvimento de uma ferramenta para Avaliação de Processos de Software baseada no Método de Avaliação MPS.BR, versão 1.0. Essa ferramenta poderá ser utilizada para as organizações se auto-avaliarem, obtendo o seu nível de maturidade, além de identificar os pontos fracos e oportunidades de melhoria do seu processo. Outra abordagem interessante é utilizá-la durante a avaliação oficial MPS.BR. Os avaliadores poderiam utilizar a ferramenta como suporte ao processo de avaliação.

Atualmente, os avaliadores MPS.BR utilizam uma planilha excel para cadastrar as evidências e atribuir os conceitos aos processos avaliados. O uso de uma ferramenta automatizaria o processo de avaliação e permitiria a geração dos relatórios finais automaticamente. Se as organizações utilizassem a ferramenta ProEvaluator para avaliações internas, formariam uma base histórica digital de avaliações. Isso permitiria que consultas fossem feitas para observar tendências. Por exemplo, poder-se-ia constatar que a maioria dos projetos da organização tem conceitos negativos em Gestão de Projetos, ou seja, elas podem estar tendo dificuldade de gerir seus projetos. Dada essa informação, ações poderiam ser tomadas para resolver os problemas identificados.

A ferramenta ProEvaluator foi analisada por profissionais da área de Engenharia de Software e pessoas certificadas no modelo MPS.BR. Segundo os resultados dessa avaliação, no estágio atual da ferramenta ProEvaluator, ela é mais adequada para ser utilizada numa pré-avaliação MPS.BR. Melhorias na ferramenta, como, por exemplo, torná-la uma ferramenta multi-usuário, deixá-la com uma interface WEB, extendê-la para contemplar a fase de planejamento da avaliação, entre outras, permitirão que ela seja utilizada numa avaliação oficial MPS.BR.

\section{Referências}

Appraisal Requirements for CMMI (2001), Version 1.1 (ARC, V1.1), Pittsburgh, Software Engineering Institute, Carnegie Mellon University. Disponível em: http://www.sei.cmu.edu 
ASSOCIAÇÃO PARA PROMOÇÃO DA EXCELÊNCIA DO SOFTWARE BRASILEIRO - SOFTEX. MPS.BR (2006), “Guia Geral”, versão 1.1. Disponível em: www.softex.br.

ASSOCIAÇÃO PARA PROMOÇÃO DA EXCELÊNCIA DO SOFTWARE BRASILEIRO - SOFTEX. MPS.BR (2006), "Guia de Avaliação", versão 1.0. Disponível em: www.softex.br.

Capability Maturity Model Integration (2002), Version 1.1 CMMI for Software Engineering (CMMI-SW, V1.1), Pittsburgh, Software Engineering Institute, Carnegie Mellon University. Disponível em: http://www.sei.cmu.edu

Capability Maturity Model for Software (1993), Version 1.1, Pittsburg, Software Engineering Institute, Carnigie Mellon University. Disponível em: http://www.sei.cmu.edu

Fabbrini, F., Fantini, E., Fusani, M., Lami, G. (2003), "Performing SPICE Assessments: Yet Another Tool". In Joint ESA - 3rd International SPICE Conference on Process Assessment and Improvement 17-21 ESTEC, Noordwijk, The Netherlands.

Galotta, C., Oliveira, K., Rocha, A.R. (2004), “Apoio a Interação entre Processos de Negócio e de Software através de Gerência do Conhecimento”, Simpósio Brasileiro de Qualidade de Software, Brasília-DF, Brasil.

Hunter, R., Robinson, G., Woodman, I. (1997), “Tool Support for Software Process Assessment and Improvement". University of Strathclyde, Department of Computer Science. Disponível em: https://www.interscience.wily.com

Muradas, F.M. (2006), "Processo de Avaliação MPS.BR: Definição e Ambiente de Apoio", COPPE/UFRJ, Rio de Janeiro.

NBR/ISO 9000 (2000), "Sistema de Gestão da Qualidade - Fundamentos e Vocabulário", Associação Brasileira de Normas Técnicas, Rio de Janeiro, Brasil.

Standard CMMI Appraisal Method for Process Improvement - SCAMPI (2002), Version 1.1, Method Implementation Guidance for Government Source and Contract Process Monitoring, Pittsburgh, Software Engineering Institute, Carnegie Mellon University. Disponível em: http://www.sei.cmu.edu

The International Organization for Standardization and the International Electrotechnical Commission. ISO/IEC 15504-5: Information Technology - Process Assessment - Part 5: An exemplar Process Assessment Model, Geneve: ISO, 2006.

The International Organization for Standardization and the International Electrotechnical Commission (1995). ISO/IEC 12207 Information technology Software life cycle processes, Geneve: ISO.

Walker, A.J., Lok, H.R. (1995), "SPICE Assessments using the SEAL assessment tool", Software Engineering Applications Laboratory. 